\title{
SACAROSE NO LABORATÓRIO DE QUíMICA ORGÂNICA DE GRADUAÇÃo
}

\author{
Vitor F. Ferreira* e Fernando C. Silva \\ Departamento de Química Orgânica, Instituto de Química, Universidade Federal Fluminense, Campus do Valonguinho, Outeiro de \\ São João Batista s/n, 24020-150 Niterói - RJ \\ Clarissa C. Perrone \\ Divisão de Química Orgânica, Instituto Nacional de Tecnologia, Av. Venezuela 82, 20081-310 Rio de Janeiro - RJ
}

Recebido em 22/9/00; aceito em 10/5/01

\begin{abstract}
SUCROSE IN UNDERGRADUATE ORGANIC CHEMISTRY LABORATORY. An experiment showing the readily available disaccharide (sucrose) as reagent for experiments in undergraduate chemistry laboratory is described. The preparation of 2,3:4,5-di-O-isopropylidene- $\beta$-D-fructopyranose from sucrose is very simple, uses low cost materials, requires two periods of 4 hours and is useful for classroom support in undergraduate courses.
\end{abstract}

Keywords: chemical education; carbohydrate; sucrose; fructose.

A inter-relação entre o conhecimento adquirido em química através de estudos teóricos e a experimentação laboratorial ${ }^{1}$, quando realizada de forma bem clara e coordenada permite ao aluno a construção de conceitos de forma mais sólida e com um maior relacionamento entre os tópicos teóricos. Neste sentido, o laboratório de química experimental é a grande oportunidade que os alunos têm de ter contato com o mundo real da química, que se bem conduzida, leva ao aprofundamento do aprendizado de diversos temas pouco explorados em sala de aula como, por exemplo, processos de separação e técnicas espectroscópicas ${ }^{2}$. Porém, o custo dos reagentes químicos, aliado a uma dificuldade de importação, torna a química orgânica experimental onerosa para os cursos de graduação em química, farmácia e engenharia química. A busca por melhores métodos experimentais $^{3,4}$, computacionais ${ }^{5}$ ou experimentos ricos em subsídios para discussões teóricas utilizando reagentes de baixo custo e de fácil aquisição, tem sido um tema bastante investigado nas diversas áreas da química ${ }^{6-8}$. Não obstante, ainda dispõem-se de poucos experimentos em livros e artigos ${ }^{9,10}$ com estas características em química orgânica.

Neste trabalho apresentamos um experimento simples de ser efetuado no laboratório e com considerável subsídio para discussões teóricas, a partir da sacarose (açúcar), que é um carboidrato muito comum no cotidiano de todos.

Os carboidratos existem difundidos em toda natureza e alguns existem praticamente puros, tais como: sacarose, glicose, frutose, amido e celulose, este último no algodão, madeira e papel $^{11}$. Em termos de volume de produção mundial, os carboidratos ficam atrás apenas dos óleos vegetais. Cerca de $95 \%$ da biomassa produzida na natureza é formada de carboidratos (200 bilhões de toneladas). Apesar desta enorme quantidade, apenas $3 \%$ são usadas pelo homem. O resto se decompõe e é reciclado pela natureza. Na utilização industrial, excluindo a alimentação, essa percentagem cai muito mais e seu uso é restrito a alguns mono e dissacarídeos de baixo peso molecular. Esses carboidratos de baixos pesos moleculares são extremamente atrativos como matéria-prima para experimentos laboratoriais de química, devido aos seguintes aspectos: disponibilidade em grandes quantidades, baixos custos, produtos oriundo de tecnologia renováveis e ecologicamente adequados.

A sacarose (1), um dissacarídeo conhecido desde o ano 200 A. C. ${ }^{12}$, é o carboidrato cristalino mais abundante na natureza ${ }^{13}$.

\footnotetext{
"e-mail: cegvito@vm.uff.br
}

É produzido em larga escala por diversos países (100.000 ton/ ano), principalmente o Brasil, onde custa $\mathrm{R} \$ 0,50 / \mathrm{Kg}$. A hidrólise da sacarose em meio ácido produz um mistura equimolecular de D-Glicose (aldose) e D-frutose (cetose), conhecida como açúcar invertido ${ }^{14}$, por ter a rotação ótica invertida em relação à substância 1 (Esquema 1). Devido à proporção (1:1), juntamente com sua solubilidade em água, estes dois carboidratos são de difícil separação por métodos convencionais.

No experimento proposto, a sacarose (1, açúcar comercial) é hidrolisada em meio ácido utilizando a acetona como solvente e reagente. A glicose e a frutose formadas (ver Esquema 1) no meio reacional são imediatamente convertidas em seus respectivos diacetonídeos ${ }^{15} 2$ (1,2:5,6-di- $O$-isopropilideno-a-D-glicofuranose) e 3 (2,3:4,5-di- $O$-isopropilideno- $\alpha$-D-frutopiranose $)^{16}$. Estes diacetonídeos também são de difícil separação, pois ambos são bem solúveis em solventes orgânicos e têm Rfs similares na cromatografia em camada fina. Porém, a hidrólise seletiva do acetal das posições 5 e 6 do diacetonídeo 2 leva ao monoacetonídeo 4, que é bem solúvel em água. Desta forma, é possível separar o diacetonídeo da frutose (3), que fica solubilizado no solvente orgânico (Esquema 1). Esta diferença na velocidade de hidrólise entre $\mathbf{2}$ e $\mathbf{3}$ está relacionada com tipo de acetal. Na substância 2 o acetal está localizado em cadeia lateral com carbono primário (C-6 da glicose) facilitando a hidrólise do mesmo.

De um modo geral, o mecanismo de formação de um acetal envolve uma dupla adição de um diol vicinal à acetona protonada (Esquema 2). E interessante observar que os acetonídeos são importantes derivados dos carboidratos e, particularmente, o diacetonídeo da frutose 3 tem sido utilizado pelo nosso grupo de pesquisa como auxiliar de quiralidade e catalisador quiral em síntese assimétrica ${ }^{17}$. Esta área da química é uma das fronteiras atuais da química orgânica cujo objetivo principal é a preparação de substâncias enantiomericamente puras através da síntese exclusiva ou preferencial de um estereoisômero ${ }^{18,19}$.

Dependendo do material que está sendo explorado na parte teórica, este experimento pode ser utilizado para ilustrar os seguintes temas: a) o mecanismo de hidrólise de dissacarídeos (tema: carboidratos); b) o mecanismo de formação de acetal e sua cinética de hidrólise (tema: reação de acetalização de aldeído e cetona), c) a separação por diferença de solubilidade (tema: técnicas de separação), d) mostrar no ensino graduação que carboidratos são substâncias vinculadas aos álcoois, aldeídos e cetonas e e) uso de grupos protetores em química orgânica. Deve-se ressaltar que um grupo protetor ${ }^{20}$ é um grupo destinado a bloquear um determinado 


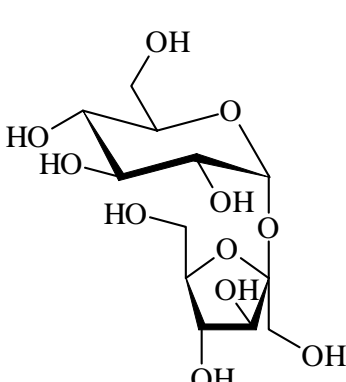

1

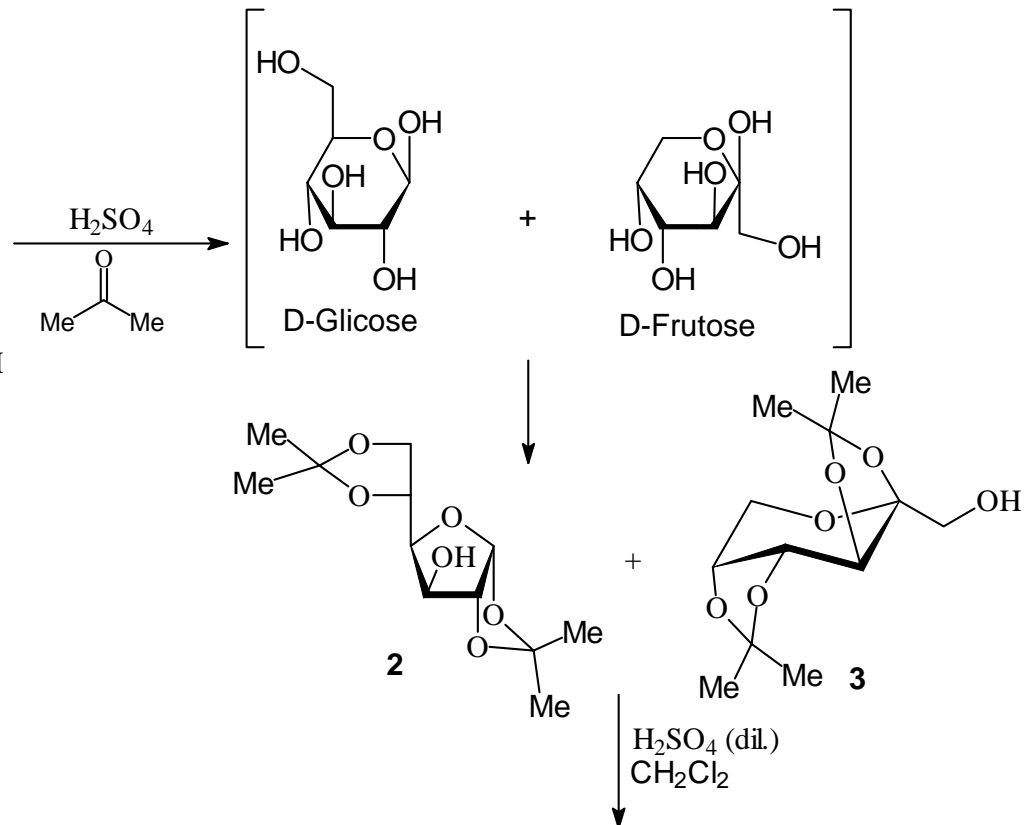

3

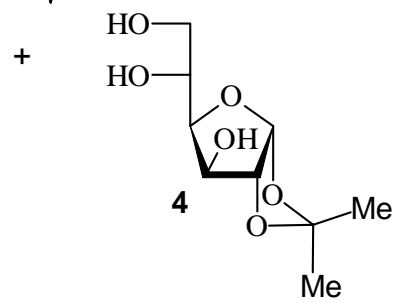

Esquema 1. Obtenção do diacetonídeo 3 a partir da sacarose (1).<smiles>CC(=O)[O+]=O</smiles><smiles>O[C@H]1C[C@@H](O)[C@H]1O</smiles><smiles>C[C@@H]1C[C@H](O)[C@H](OC(C)(C)O)C1</smiles><smiles>CC(C)(C)[C@@H]1CC[C@@H](O)[C@@H](O)C1</smiles>

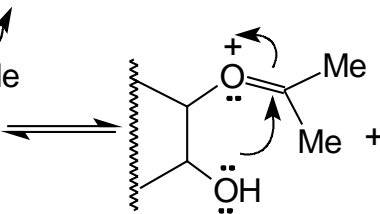<smiles></smiles><smiles>CC1(C)C[C@H]2C[C@H](O)[C@@H](C2)O1</smiles><smiles>[Os][Os]</smiles><smiles>CC1(C)O[C@@H]2C[C@H](O1)[C@H](O)C2</smiles><smiles>[C+][CH+]</smiles>

Esquema 2. Mecanismo geral de formação de acetonídeos.

grupo funcional contra reações indesejadas em uma determinada etapa. Normalmente este grupo é removido em etapas posteriores. O uso desta estratégia em síntese orgânica introduz duas etapas a mais na síntese e, portanto seu uso deve ser evitado ao extremo. Um grupo de proteção para ser considerado ideal deve ser estável frente a várias condições experimentais e de fácil remoção em condições brandas.

Em resumo, este é um experimento simples de ser realizado em dois períodos de $4 \mathrm{~h}$ de laboratório, utiliza materiais de baixo custo, as vidrarias utilizadas e equipamentos são comuns em todos os laboratórios e produz a substância 3 que é de fácil cristalização.

\section{PROCEDIMENTO EXPERIMENTAL}

\section{$\mathbf{2 , 3 ; 4 , 5 - d i - O - i s o p r o p i l i d e n o - \beta - D - f r u t o p i r a n o s e ~ ( 3 ) ~}$}

Em erlenmeyer de $500 \mathrm{~mL}$, uma mistura de açúcar comercial (15 g, 44,8 mmol) e acetona $(200 \mathrm{~mL})$ é resfriada externamente com gelo até cerca de $5{ }^{\circ} \mathrm{C}$ e agitada vigorosamente (pode utilizar agitador mecânico ou magnético). A seguir é adicionado lentamente ácido sulfúrico concentrado $(8 \mathrm{~mL})$. Em seguida a mistura é adicionalmente agitada por mais $2 \mathrm{~h}$ e 30 min à temperatura ambiente. $\mathrm{O}$ meio reacional é então resfriado até atingir uma temperatura entre $0-10{ }^{\circ} \mathrm{C}$ e neutralizado 
com $\mathrm{NaOH} 50 \%$ p/v. O pH neutro final da solução deve ser controlado com a adição de solução saturada de $\mathrm{Na}_{2} \mathrm{CO}_{3}$. Os sólidos $\left(\mathrm{Na}_{2} \mathrm{SO}_{4}\right.$ e sacarose não reagida) são removidos por filtração em papel. A solução é evaporada sob pressão reduzida (a temperatura do banho não deve ultrapassar os $\left.55^{\circ} \mathrm{C}\right)^{21}$, obtendo-se um xarope amarelo claro contendo a mistura dos diacetonídeos 2 e 3. Pode-se interromper o experimento na solução filtrada ou no xarope, que devem ser estocados no refrigerador até a próxima aula.

Em erlenmeyer de $500 \mathrm{~mL}$ o xarope anterior é dissolvido em diclorometano $(200 \mathrm{~mL})$ e adicionado $200 \mathrm{~mL}$ de uma solução de $\mathrm{H}_{2} \mathrm{SO}_{4}(0,5 \mathrm{~mol} / \mathrm{L})$. A mistura é fortemente agitada durante 2 horas (não se deve ultrapassar esse tempo), a fase orgânica é separada, lavada com solução saturada de $\mathrm{NaHCO}_{3}$ $(1 \times 50 \mathrm{~mL})$, com água $(4 \times 50 \mathrm{~mL})$ e seca com sulfato de sódio anidro. Em seguida, o solvente é evaporado (pode ser sob pressão reduzida) até obtenção de um sólido branco, o qual é recristalizado em hexano, com filtração à quente em carvão ativo. O produto 3 foi obtido $(3,8 \mathrm{~g})$ sob forma de agulhas finas (p.f $97{ }^{\circ} \mathrm{C}$, lit. $95-96{ }^{\circ} \mathrm{C}$ ) ${ }^{22}$ em $33 \%$ de rendimento.

(3): I.V. $v_{\max }\left(\mathrm{KBr}, \mathrm{cm}^{-1}\right): 3290(\mathrm{OH}) ; 1380 ; 1370$ (metilas geminadas); RMN de ${ }^{1} \mathrm{H}\left(200 \mathrm{MHz}, \mathrm{CDCl}_{3}, \delta\right): 1,35(\mathrm{~s}, 3 \mathrm{H}$, $\left.\mathrm{CH}_{3}\right) ; 1,39\left(\mathrm{~s}, 3 \mathrm{H}, \mathrm{CH}_{3}\right) ; 1,47\left(\mathrm{~s}, 3 \mathrm{H}, \mathrm{CH}_{3}\right) ; 1,55\left(\mathrm{~s}, 3 \mathrm{H}, \mathrm{CH}_{3}\right)$; 2,13 (ddd, $J=0,6, J=5,3, J=7,8 \mathrm{~Hz}, 1 \mathrm{H}, \mathrm{OH}$ ); 3,64 - 3,72 (sm, $2 \mathrm{H}, \mathrm{H}_{1}$ e $\mathrm{H}_{1}$ ) ; 3,77 (dd, $J=0,7, J=13,1 \mathrm{~Hz}, 1 \mathrm{H}, \mathrm{H}_{6}$ ) ; $3,92\left(\mathrm{dd}, J=2,1, J=13,1 \mathrm{~Hz}, 1 \mathrm{H}, \mathrm{H}_{6}\right) ; 4,24$ (ddd, $J=8,0$, $\left.J=2,1, J=0,7 \mathrm{~Hz}, 1 \mathrm{H}, \mathrm{H}_{5}\right) ; 4,34\left(\mathrm{~d}, J=2,8,1 \mathrm{H}, \mathrm{H}_{3}\right) ; 4,61$ $\left(\mathrm{dd}, J=2,8, J=8,0 \mathrm{~Hz}, 1 \mathrm{H}, \mathrm{H}_{4}\right)$.

\section{AGRADECIMENTOS}

Ao $\mathrm{CNPq}$ pela bolsa de iniciação científica concedida ao aluno Fernando C. da Silva. A UFF, INT e a FAPERJ pelos recursos financeiros. Especiais agradecimentos ao Prof. João Augusto de M. Gouveia-Matos (IQ-UFRJ) pelas valiosas sugestões na elaboração deste texto.

\section{REFERÊNCIAS}

1. Hamelin; R.; Quim. Nova 1995, 18, 68.

2. Bieber, L. W.; Quim. Nova 1999, 22, 605.

3. Imamura, P. M.; Baptistella, L. H. B.; Quim. Nova 2000, 23, 270.

4. Reis; C.; Andrade, J. C.; Quim. Nova 1996, 19, 313.
5. Machado, B. C.; Machado, S. P.; Herrera, J. O. M.; Quim. Nova 1992, 15, 250.

6. Pereira, M. P. B. A.; Quim. Nova 1989, 12, 182.

7. Soares, B. G.; Pires, D. X.; Souza, N. A.; Quim. Nova 1987, 10, 27.

8. Vianna, J. F.; Sleet, R. J.; Jonhstone, A. H.; Quim. Nova 1999, 22, 280.

9. Ferreira, V. F.; Quim. Nova na Escola 1996, 35.

10. Cunha, A. C.; Pereira, L. O. R.; de Souza, M.C.B.V.; Ferreira, V.F.; J. Chem. Educ. 1999, 76, 79.

11. Ferreira, V.F.; Quim. Nova 1995, 18, 267.

12. Rossiter, B. T.; Swingle, M. M.; Chem. Rev. 1992, 92, 771.

13. Khan, R.; Pure Appl. Chem. 1984, 56, 833.

14. Snyder, C. H.; The Extraordinary Chemistry of Ordinary Things; 2nd Ed. John Wiley \& Sons, USA, 1995, p. $439-446$.

15. a) Acetonídeos são substâncias derivadas da reação entre dióis, preferencialmente vicinais, e acetona catalisada por ácido. b) de Belder, A. N.; Adv. Carbohydr. Chem. Biochem. 1965, 20, 219. c) Brady Jr, R. F.; Adv. Carbohydr. Chem. Biochem. 1971, 26, 197. d) Brady Jr, R. F.; Carbohydr. Chem. 1979, 15, 35. e) Wolfrom, M. L.; Diwadka, Gelas, Horton, D.; Carbohydr. Chem. 1974, 35, 87 .

16. Fischer, E.; Ber. 1885, 28, 1145.

17. a) Ferreira, V. F.; Costa, P. R. R.; Pinheiro, S.; Filho, H. C. A.; J. Braz. Chem. Soc. 1996, 7, 67. b) Pinheiro, S.; Pedraza, S. F.; Peralta, M. A.; Carvalho, E. M.; Farias, F. M. C.; Ferreira, V. F.; J. Carbohydr. Chem. 1998, 17, 901. c) Ferreira, M. L. G.; Pinheiro, S.; Ferreira, V. F.; Perrone, C. C.; Costa, P. R. R.; Tetrahedron: Asymmetry 1998, 9, 2671. d) Ferreira, V. F.; Perrone, C. C.; Pinheiro, S.; Costa, P. R. R.; J. Braz. Chem. Soc. 2000, 11, 266.

18. S. Pinheiro; V. F. Ferreira; Quim. Nova 1998, 21, 312.

19. Ferreira, V. F.; Barreiro, E. L. J.; Costa, P. R. R.; Quim. Nova 1997, 20, 647.

20. a) Greene, T. W.; Protective Group in Organic Synthesis; Wiley, NY, 1981. b) Schelhaas, G.; Waldmann, H.; Angew. Chem. Int. Ed. Engl. 1996, 35, 2056.

21. Esta evaporação pode ser realizada com bomba de vácuo do tipo trompa d'água.

22. a) Bell, D. J.; J. Chem. Soc. 1947, 1461. b) Chittenden, G. J. F.; Verhart, C. C. J.; Carls, M. G. C.; Zwanenburg, B.; Recl. Trav. Chim. Pays-Bas 1992, 11, 348. 